\title{
Directional droplet transport mediated by circular groove arrays, Part I: Experimental findings
}

Cong Liu ${ }^{1}$, Irina Legchenkova ${ }^{2}$, Libao Han ${ }^{1}$, Wenna $\mathrm{Ge}^{1}$, Cunjing $\mathrm{Lv}^{3}$, Shile Feng ${ }^{1}$, Edward Bormashenko ${ }^{2 *}$, Yahua Liu ${ }^{1 *}$

${ }^{1}$ Key Laboratory for Precision and Non-traditional Machining Technology of Ministry of Education, Dalian University of Technology, Dalian 116024, China

${ }^{2}$ Chemical Engineering Department, Engineering Faculty, Ariel University, Ariel, 40700, Israel

${ }^{3}$ Department of Engineering Mechanics, Tsinghua University, Beijing 100084, China

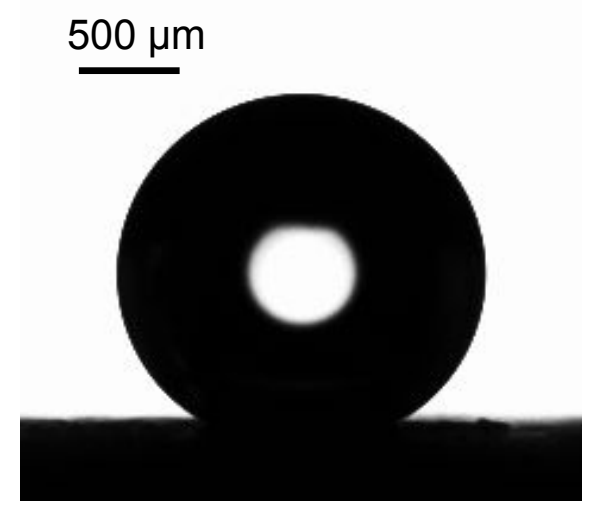

Figure S1. Contact angle on circular equidistant grooves (with $W=100 \mu \mathrm{m}, S=450$ $\mu \mathrm{m}, H=350 \mu \mathrm{m})$ decorated with hydrophobic $\mathrm{ZnO}$ nanoparticles. The apparent contact angle of the surface at $r=0 \mathrm{~mm}$ is $160 \pm 2.1^{\circ}$ and the contact angle hysteresis is $\sim 5^{\circ}$.

Movie S1. The side and top views of a droplet impacting on circular groove arrays with deviation distance between impact point and center of curvature $r=0 \mathrm{~mm}$.

Movie S2. The side and top views of a droplet impacting on circular groove arrays with deviation distance between impact point and center of curvature $r=2.1 \mathrm{~mm}$. 